\title{
Integrated Working Fluids and Process Optimization for Refrigeration Systems Using Polar PC-SAFT
}

\author{
Jiayuan Wang, Duoduo Chen, Lingyu Zhu* \\ College of Chemical Engineering, Zhejiang University of Technology, 310014, \\ Hangzhou, China
}

Email: zhuly@zjut.edu.cn 


\section{Supporting information}

\section{The Polar PC-SAFT parameters}

Table S1. A summary of Polar PC-SAFT parameters for 39 refrigerants.

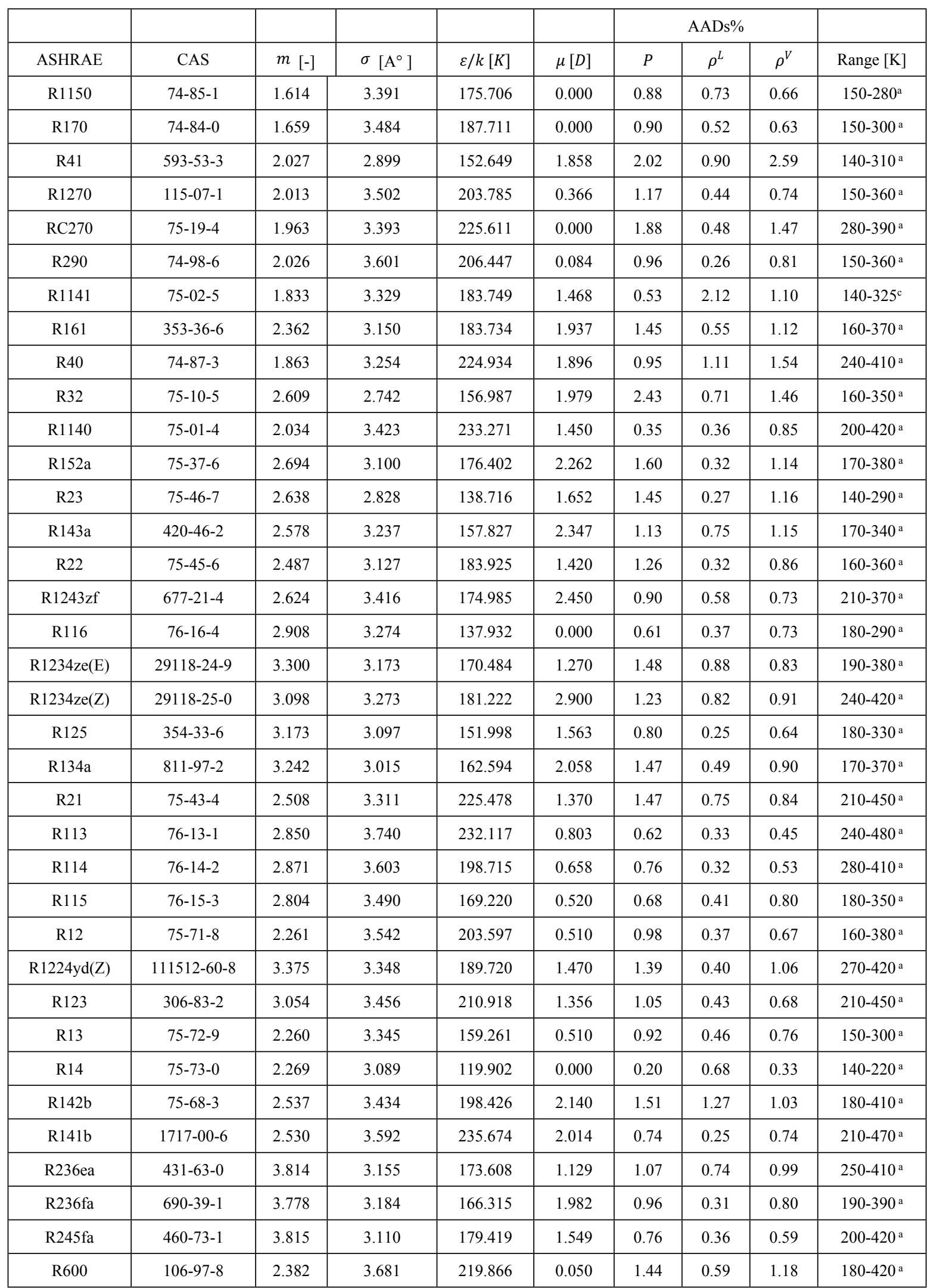




\begin{tabular}{|c|c|c|c|c|c|c|c|c|c|}
\hline R1234yf & $754-12-1$ & 2.744 & 3.402 & 167.062 & 2.480 & 0.64 & 0.76 & 0.59 & $170-360^{\mathrm{a}}$ \\
\hline R1123 & $359-11-5$ & 3.017 & 2.896 & 150.789 & 1.400 & 2.15 & 0.85 & 1.77 & $210-330^{\mathrm{a}}$ \\
\hline R150 & $107-06-2$ & 2.755 & 3.380 & 273.441 & 1.440 & 2.03 & 1.58 & 1.99 & $260-560^{\mathrm{a}}$ \\
\hline
\end{tabular}

a.REFPROP 10.0; ${ }^{\mathrm{b}}$ CRC Handbook of Chemistry and Physics; ${ }^{\mathrm{c}}$ ASPEN NIST ThermoData Engine.

\section{The Polar PC-SAFT equation of state}

\section{Residual Helmholtz free energy}

$$
\begin{aligned}
& \tilde{a}=\frac{A}{N k T} \\
& \tilde{a}^{\text {res }}=\tilde{a}^{\text {chain }}+\tilde{a}^{\text {disp }}+\tilde{a}^{\text {polar }} \\
& \tilde{a}^{\text {chain }}=\bar{m} \tilde{a}^{h s}-\sum_{i} x_{i}\left(m_{i}-1\right) \operatorname{lng} g_{i i}^{h s}\left(\sigma_{i i}\right)
\end{aligned}
$$

$\bar{m}=\sum_{i} x_{i} m_{i}$

$\tilde{a}^{h s}=\frac{1}{\zeta_{0}}\left[\frac{3 \zeta_{1} \zeta_{2}}{\left(1-\zeta_{3}\right)}+\frac{\zeta_{2}^{3}}{\zeta_{3}\left(1-\zeta_{3}\right)^{2}}+\left(\frac{\zeta_{2}^{3}}{\zeta_{3}^{2}}-\zeta_{0}\right) \ln \left(1-\zeta_{3}\right)\right]$

$g_{i j}^{h s}=\frac{1}{\left(1-\zeta_{3}\right)}+\left(\frac{d_{i} d_{j}}{d_{i}+d_{j}}\right) \frac{3 \zeta_{2}}{\left(1-\zeta_{3}\right)^{2}}+\left(\frac{d_{i} d_{j}}{d_{i}+d_{j}}\right)^{2} \frac{2 \zeta_{2}^{2}}{\left(1-\zeta_{3}\right)^{3}}$

$\zeta_{n}=\frac{\pi}{6} \rho \sum_{i} x_{i} m_{i} d_{i}^{n} \quad n \in\{0,1,2,3\}$

$d_{i}=\sigma_{i}\left[1-0.12 \exp \left(-3 \frac{\epsilon_{i}}{k T}\right)\right]$

$\tilde{a}^{d i s p}=-2 \pi \rho I_{1}(\eta, \bar{m}) \overline{m^{2} \epsilon \sigma^{3}}-\pi \rho \bar{m} C_{1} I_{2}(\eta, \bar{m}) \overline{m^{2} \epsilon^{2} \sigma^{3}}$ 


$$
\begin{aligned}
& C_{1}=\left(1+Z^{h c}+\rho \frac{\partial Z^{h c}}{\partial \rho}\right)^{-1} \\
& =\left(1+\bar{m} \frac{8 \eta-2 \eta^{2}}{(1-\eta)^{4}}+(1-\bar{m}) \frac{20 \eta-27 \eta^{2}+12 \eta^{3}-2 \eta^{4}}{[(1-\eta)(2-\eta)]^{2}}\right)^{-1} \\
& \overline{m^{2} \in \sigma^{3}}=\sum_{i} \sum_{j} x_{i} x_{j} m_{i} m_{j}\left(\frac{\epsilon_{i j}}{k T}\right) \sigma_{i j}^{3} \\
& \overline{m^{2} \epsilon^{2} \sigma^{3}}=\sum_{i} \sum_{j} x_{i} x_{j} m_{i} m_{j}\left(\frac{\epsilon_{i j}}{k T}\right)^{2} \sigma_{i j}^{3} \\
& \sigma_{i j}=\frac{1}{2}\left(\sigma_{i}+\sigma_{j}\right) \\
& \epsilon_{i j}=\sqrt{\epsilon_{i} \epsilon_{j}} \\
& I_{1}(\eta, \bar{m})=\sum_{i=0}^{6} a_{i}(\bar{m}) \eta^{i} \\
& I_{2}(\eta, \bar{m})=\sum_{i=0}^{6} b_{i}(\bar{m}) \eta^{i} \\
& a_{i}(\bar{m})=a_{0 i}+\frac{\bar{m}-1}{\bar{m}} a_{1 i}+\frac{\bar{m}-1 \bar{m}-2}{\bar{m} \bar{m}} a_{2 i} \\
& b_{i}(\bar{m})=b_{0 i}+\frac{\bar{m}-1}{\bar{m}} b_{1 i}+\frac{\bar{m}-1 \bar{m}-2}{\bar{m} \bar{m}} b_{2 i} \\
& \tilde{a}^{\text {polar }}=\frac{\tilde{a}_{2}}{1-\tilde{a}_{3} / \tilde{a}_{2}} \\
& \tilde{a}_{2}=-\pi \rho \sum_{i} \sum_{j} x_{i} x_{j} \frac{\epsilon_{i i} \epsilon_{j j} \sigma_{i i}^{3} \sigma_{j j}^{3}}{k T k T \sigma_{i j}^{3}} n_{p i} n_{p j} \mu_{i}^{* 2} \mu_{j}^{* 2} I_{2, i j}
\end{aligned}
$$




$$
\begin{aligned}
& \tilde{a}_{3}=-\frac{4 \pi^{2}}{3} \rho^{2} \sum_{i} \sum_{j} \sum_{k} x_{i} x_{j} x_{k} \frac{\epsilon_{i i} \epsilon_{j j} \epsilon_{k k} \sigma_{i i}^{3} \sigma_{j j}^{3} \sigma_{k k}^{3} T k \sigma_{i j} \sigma_{i k} \sigma_{j k}}{k i} n_{p j} n_{p k} \mu_{i}^{* 2} \mu_{j}^{* 2} \mu_{k}^{* 2} I_{3,} \\
& I_{2, i j}=\sum_{n=0}^{4}\left(a_{n, i j}+b_{n, i j} \frac{\epsilon_{i j}}{k T}\right) \eta^{n} \\
& I_{3, i j k}=\sum_{n=0}^{4} c_{n, i j k} \eta^{n} \\
& a_{n, i j}=a_{0 n}+\frac{m_{i j}-1}{m_{i j}} a_{1 n}+\frac{m_{i j}-1 m_{i j}-2}{m_{i j}} m_{i j} \\
& \mu_{2 n}^{* 2}=\mu_{i}^{2} /\left(m_{i} \epsilon_{i i} \sigma_{i i}^{3}\right) \\
& m_{n, i j}=b_{0 n}+\frac{m_{i j}-1}{m_{i j}} b_{1 n}+\frac{m_{i j}-1 m_{i j}-2}{m_{i j}} b_{2 n} \\
& m_{i j}=\left(m_{i} m_{j}\right)^{1 / 2} w i t h m_{i j} \leq 2 \\
& c_{n, i j k}=c_{0 n}+\frac{m_{i j k}-1}{m_{i j k}} c_{1 n}+\frac{m_{i j k}-1 m_{i j k}-2}{m_{i j k}} m_{i j k} \\
& \mu_{2 n}
\end{aligned}
$$

\section{Compressibility factor}

$\mathrm{Z}=1+\rho\left(\frac{\partial \tilde{a}^{r e s}}{\partial \rho}\right)_{T, x_{i}}$

$\mathrm{Z}=1+Z^{\text {chain }}+Z^{\text {disp }}+Z^{\text {polar }}$

$Z^{\text {chain }}=\rho\left(\frac{\partial \tilde{a}^{\text {chain }}}{\partial \rho}\right)_{T, x_{i}}=\bar{m} Z^{h s}-\sum_{i} x_{i}\left(m_{i}-1\right)\left(g_{i i}^{h s}\right)^{-1} \rho \frac{\partial g_{i i}^{h s}}{\partial \rho}$ 


$$
Z^{h s}=\frac{\zeta_{3}}{\left(1-\zeta_{3}\right)}+\frac{3 \zeta_{1} \zeta_{2}}{\zeta_{0}\left(1-\zeta_{3}\right)^{2}}+\frac{3 \zeta_{2}^{3}-\zeta_{3} \zeta_{2}^{3}}{\zeta_{0}\left(1-\zeta_{3}\right)^{3}}
$$

$\rho \frac{\partial g_{i j}^{h s}}{\partial \rho}$

$$
\begin{aligned}
& =\frac{\zeta_{3}}{\left(1-\zeta_{3}\right)^{2}}+\left(\frac{d_{i} d_{j}}{d_{i}+d_{j}}\right)\left(\frac{3 \zeta_{2}}{\left(1-\zeta_{3}\right)^{2}}+\frac{6 \zeta_{2} \zeta_{3}}{\left(1-\zeta_{3}\right)^{3}}\right)+\left(\frac{d_{i} d_{j}}{d_{i}+d_{j}}\right)^{2} \\
& \left(\frac{4 \zeta_{2}^{2}}{\left(1-\zeta_{3}\right)^{3}}+\frac{6 \zeta_{2}^{2} \zeta_{3}}{\left(1-\zeta_{3}\right)^{4}}\right)
\end{aligned}
$$

$Z^{\text {disp }}=\rho\left(\frac{\partial \tilde{a}^{d i s p}}{\partial \rho}\right)_{T, x_{i}}=-2 \pi \rho \frac{\partial\left(\eta I_{1}\right)}{\partial \eta} \overline{m^{2} \epsilon \sigma^{3}}-\pi \rho \bar{m}\left[C_{1} \frac{\partial\left(\eta I_{2}\right)}{\partial \eta}+C_{2} \eta I_{2}\right] \overline{m^{2} \epsilon^{2} \sigma^{3}}(\mathrm{~S} 35$

$\frac{\partial\left(\eta I_{1}\right)}{\partial \eta}=\sum_{j=0}^{6} a_{j}(\bar{m})(j+1) \eta^{j}$

$\frac{\partial\left(\eta I_{2}\right)}{\partial \eta}=\sum_{j=0}^{6} b_{j}(\bar{m})(j+1) \eta^{j}$

$C_{2}=\frac{\partial C_{1}}{\partial \eta}=-C_{1}^{2}\left(\bar{m} \frac{-4 \eta^{2}+20 \eta+8}{(1-\eta)^{5}}+(1-\bar{m}) \frac{2 \eta^{3}+12 \eta^{2}-48 \eta+40}{[(1-\eta)(2-\eta)]^{3}}\right)$

$Z^{\text {polar }}=\rho\left(\frac{\partial \tilde{a}^{\text {polar }}}{\partial \rho}\right)_{T, x_{i}}=\rho \frac{\tilde{a}_{2}\left(\tilde{a}_{2, \rho}+\tilde{a}_{3, \rho}\right)-2 \tilde{a}_{2, \rho} \tilde{a}_{3}}{\tilde{a}_{2}\left(1-\tilde{a}_{3} / \tilde{a}_{2}\right)^{2}}$

$\tilde{a}_{2, \rho}=-\pi \sum_{i} \sum_{j} x_{i} x_{j} \frac{\epsilon_{i i} \epsilon_{j j} \sigma_{i i}^{3} \sigma_{j j}^{3}}{k T k T \sigma_{i j}^{3}} n_{p i} n_{p j} \mu_{i}^{* 2} \mu_{j}^{* 2}\left(I_{2, i j}+\rho \frac{\partial I_{2, i j}}{\partial \rho}\right)$

$\rho \frac{\partial I_{2, i j}}{\partial \rho}=\sum_{n=0}^{4} n\left(a_{n, i j}+b_{n, i j} \frac{\epsilon_{i j}}{k T}\right) \eta^{n}$ 
$\tilde{a}_{3, \rho}$

$=-\frac{4 \pi^{2} \rho}{3}$

$\sum_{i} \sum_{j} \sum_{k} x_{i} x_{j} x_{k} \frac{\epsilon_{i i} \epsilon_{j j} \epsilon_{k k} \sigma_{i i}^{3} \sigma_{j j}^{3} \sigma_{k k}^{3}}{k T k T T \sigma_{i j} \sigma_{i k} \sigma_{j k}} n_{p i} n_{p j} n_{p k} \mu_{i}^{* 2} \mu_{j}^{* 2} \mu_{k}^{* 2}\left(2 I_{3, i j k}+\rho \frac{\partial I_{3, i j k}}{\partial \rho}\right)$

$\rho \frac{\partial I_{3, i j k}}{\partial \rho}=\sum_{n=0}^{4} n c_{n, i j k} \eta^{n}$

\section{Fugacity coefficient}

$\ln \varphi_{k}=\frac{\mu_{k}^{r e s}(T, v)}{k T}-\ln Z$

$\frac{\mu_{k}^{r e s}(T, v)}{k T}=\tilde{a}^{r e s}+(Z-1)+\left(\frac{\partial \tilde{a}^{r e s}}{\partial x_{k}}\right)_{T, \rho, x_{j \neq k}}-\sum_{j=1}^{N}\left[x_{j}\left(\frac{\partial \tilde{a}^{r e s}}{\partial x_{j}}\right)_{T, \rho, x_{i \neq j}}\right]$

$\left(\frac{\partial \tilde{a}^{r e s}}{\partial x_{k}}\right)_{T, \rho, x_{j \neq k}}=\left(\frac{\partial \tilde{a}^{\text {chain }}}{\partial x_{k}}\right)_{T, \rho, x_{j \neq k}}+\left(\frac{\partial \tilde{a}^{\text {disp }}}{\partial x_{k}}\right)_{T, v, x_{j \neq k}}+\left(\frac{\partial \tilde{a}^{\text {polar }}}{\partial x_{k}}\right)_{T, v, x_{j \neq k}}$

$\zeta_{n, x_{k}}=\left(\frac{\partial \zeta_{n}}{\partial x_{k}}\right)_{T, \rho, x_{j \neq k}}=\frac{\pi}{6} \rho m_{k} d_{k}^{n} \quad n \in\{0,1,2,3\}$

$\left(\frac{\partial \tilde{a}^{\text {chain }}}{\partial x_{k}}\right)_{T, \rho, x_{j \neq k}}=m_{k} \tilde{a}^{h s}+\bar{m}\left(\frac{\partial \tilde{a}^{h s}}{\partial x_{k}}\right)_{T, \rho, x_{j \neq k}}-\left(m_{k}-1\right) \ln \left(g_{k k}^{h s}\right)$

$$
-\sum_{i} x_{i}\left(m_{i}-1\right)\left(g_{i i}^{h s}\right)^{-1}\left(\frac{\partial g_{i i}^{h s}}{\partial x_{k}}\right)_{T, \rho, x_{j \neq k}}
$$




$$
\begin{aligned}
\left(\frac{\partial \tilde{a}^{h s}}{\partial x_{k}}\right)_{T, \rho, x_{j \neq k}} & =-\frac{\zeta_{0, x_{k}}}{\zeta_{0}} \tilde{a}^{h s}+\frac{1}{\zeta_{0}}\left[\frac{3\left(\zeta_{1, x_{k}} \zeta_{2}+\zeta_{1} \zeta_{2, x_{k}}\right)}{\left(1-\zeta_{3}\right)}+\frac{3 \zeta_{1} \zeta_{2} \zeta_{3, x_{k}}}{\left(1-\zeta_{3}\right)^{2}}+\frac{3 \zeta_{2}^{2} \zeta_{2, x_{k}}}{\zeta_{3}\left(1-\zeta_{3}\right)}\right. \\
& +\frac{\zeta_{2}^{3} \zeta_{3, x_{k}}\left(3 \zeta_{3}-1\right)}{\zeta_{3}^{2}\left(1-\zeta_{3}\right)^{3}}+\left(\frac{3 \zeta_{2}^{2} \zeta_{2, x_{k}} \zeta_{3}-2 \zeta_{2}^{3} \zeta_{3, x_{k}}}{\zeta_{3}^{3}}-\zeta_{0, x_{k}}\right) \ln \left(1-\zeta_{3}\right) \\
& \left.+\left(\zeta_{0}-\frac{\zeta_{2}^{3}}{\zeta_{3}^{2}}\right) \frac{\zeta_{3, x_{k}}}{\left(1-\zeta_{3}\right)}\right]
\end{aligned}
$$

$$
\begin{aligned}
\left(\frac{\partial g_{i j}^{h s}}{\partial x_{k}}\right)_{T, \rho, x_{j \neq k}} & \\
& =\frac{\zeta_{3, x_{k}}}{\left(1-\zeta_{3}\right)^{2}}+\left(\frac{d_{i} d_{j}}{d_{i}+d_{j}}\right)\left(\frac{3 \zeta_{2, x_{k}}}{\left(1-\zeta_{3}\right)^{2}}+\frac{6 \zeta_{2} \zeta_{3, x_{k}}}{\left(1-\zeta_{3}\right)^{3}}\right)+\left(\frac{d_{i} d_{j}}{d_{i}+d_{j}}\right)^{2} \\
& \left(\frac{4 \zeta_{2} \zeta_{2, x_{k}}}{\left(1-\zeta_{3}\right)^{3}}+\frac{6 \zeta_{2}^{2} \zeta_{3, x_{k}}}{\left(1-\zeta_{3}\right)^{4}}\right)
\end{aligned}
$$

$\left(\frac{\partial \tilde{a}^{d i s p}}{\partial x_{k}}\right)_{T, \rho, x_{j \neq k}}$

$$
\begin{aligned}
& =-2 \pi \rho\left[I_{1, x_{k}} \overline{m^{2} \epsilon \sigma^{3}}+I_{1}\left(\overline{m^{2} \epsilon \sigma^{3}}\right)_{x_{k}}\right]-\pi \rho \\
& \left\{\left[m_{k} C_{1} I_{2}+\bar{m} C_{1, x_{k}} I_{2}+\bar{m} C_{1} I_{2, x_{k}}\right] \overline{m^{2} \epsilon^{2} \sigma^{3}}+\bar{m} C_{1} I_{2}\left(\overline{m^{2} \epsilon^{2} \sigma^{3}}\right)_{x_{k}}\right\}
\end{aligned}
$$

$$
\left(\overline{m^{2} \in \sigma^{3}}\right)_{x_{k}}=2 m_{k} \sum_{j} x_{j} m_{j}\left(\frac{\epsilon_{k j}}{k T}\right) \sigma_{k j}^{3}
$$

$$
\left(\overline{m^{2} \epsilon^{2} \sigma^{3}}\right)_{x_{k}}=2 m_{k} \sum_{j} x_{j} m_{j}\left(\frac{\epsilon_{k j}}{k T}\right)^{2} \sigma_{k j}^{3}
$$

$$
\begin{aligned}
C_{1, x_{k}} & =C_{2} \zeta_{3, x_{k}}-C_{1}^{2}\left(m_{k} \frac{8 \eta-2 \eta^{2}}{(1-\eta)^{4}}-m_{k} \frac{20 \eta-27 \eta^{2}+12 \eta^{3}-2 \eta^{4}}{[(1-\eta)(2-\eta)]^{2}}\right) \\
I_{1, x_{k}} & =\sum_{i=0}^{6}\left[a_{i}(\bar{m}) i \zeta_{3, x_{k}} \eta^{i-1}+a_{i, x_{k}} \eta^{i}\right]
\end{aligned}
$$




$$
\begin{aligned}
& I_{2, x_{k}}=\sum_{i=0}^{6}\left[b_{i}(\bar{m}) i \zeta_{3, x_{k}} \eta^{i-1}+b_{i, x_{k}} \eta^{i}\right] \\
& a_{i, x_{k}}=\frac{m_{k}}{\bar{m}^{2}} a_{1 i}+\frac{m_{k}}{\bar{m}^{2}}\left(3-\frac{4}{\bar{m}}\right) a_{2 i} \\
& b_{i, x_{k}}=\frac{m_{k}}{\bar{m}^{2}} b_{1 i}+\frac{m_{k}}{\bar{m}^{2}}\left(3-\frac{4}{\bar{m}}\right) b_{2 i} \\
& \left(\frac{\partial \tilde{a}^{\text {polar }}}{\partial x_{k}}\right)_{T, \rho, x_{j \neq k}}=\frac{\tilde{a}_{2}\left(\tilde{a}_{2, x_{k}}+\tilde{a}_{3, x_{k}}\right)-2 \tilde{a}_{2, x_{k}} \tilde{a}_{3}}{\tilde{a}_{2}\left(1-\tilde{a}_{3} / \tilde{a}_{2}\right)^{2}} \\
& \tilde{a}_{2, x_{k}} \\
& =-\pi \rho\left(2 \sum_{i} x_{i}\left(\frac{\epsilon_{i i}}{k T}\right)\left(\frac{\epsilon_{k k}}{k T}\right) \frac{\sigma_{i i}^{3} \sigma_{k k}^{3}}{\sigma_{i k}^{3}} n_{p i} n_{p k} \mu_{i}^{* 2} \mu_{k}^{* 2} I_{2, i k}+\sum_{i} \sum_{j} x_{i} x_{j}\left(\frac{\epsilon}{k}\right.\right. \\
& \left.n_{p i} n_{p j} \mu_{i}^{* 2} \mu_{j}^{* 2} I_{2, i j, x_{k}}\right) \\
& I_{2, i j, x_{k}}=\sum_{n=0}^{4} n\left(a_{n, i j}+b_{n, i j} \frac{\epsilon_{i j}}{k T}\right) \eta^{n-1} \zeta_{3, x_{k}}
\end{aligned}
$$$$
\tilde{a}_{3, x_{k}}
$$$$
=-\frac{4 \pi^{2}}{3} \rho^{2}\left(3 x_{k}^{2}\left(\frac{\epsilon_{k k}}{k T}\right)^{3} \sigma_{k k}^{6} n_{p k}^{3} \mu_{k}^{* 6} I_{3, k k k}+6 x_{k} \sum_{i \neq k} x_{i}\left(\frac{\epsilon_{i i}}{k T}\right)\left(\frac{\epsilon_{k k}}{k T}\right)^{2} \frac{\sigma_{i i}^{3} \sigma_{k l}^{5}}{\sigma_{i k}^{2}}\right.
$$$$
\mu_{k}^{* 4} I_{3, i k k}+3 \sum_{i \neq k j \neq k} x_{i} x_{j}\left(\frac{\epsilon_{i i}}{k T}\right)\left(\frac{\epsilon_{j j}}{k T}\right)\left(\frac{\epsilon_{k k}}{k T}\right) \frac{\sigma_{i i}^{3} \sigma_{j j}^{3} \sigma_{k k}^{3}}{\sigma_{i j} \sigma_{i k} \sigma_{j k}} n_{p i} n_{p j} n_{p k} \mu_{i}^{* 2} \mu_{j}^{* 2} \mu_{i}
$$$$
3, i j k+\sum_{i} \sum_{j} \sum_{g} x_{i} x_{j} x_{g}\left(\frac{\epsilon_{i i}}{k T}\right)\left(\frac{\epsilon_{j j}}{k T}\right)\left(\frac{\epsilon_{g g}}{k T}\right) \frac{\sigma_{i i}^{3} \sigma_{j j}^{3} \sigma_{g g}^{3}}{\sigma_{i j} \sigma_{i g} \sigma_{j g}} n_{p i} n_{p j} n_{p g} \mu_{i}^{* 2} \mu_{j}^{* 2} \mu_{c}
$$$$
\left.3, i j g, x_{k}\right)
$$$$
=-4 \pi^{2} \rho^{2}\left(\sum_{i \neq k j \neq k} \sum_{i} x_{j}\left(\frac{\epsilon_{i i}}{k T}\right)\left(\frac{\epsilon_{j j}}{k T}\right)\left(\frac{\epsilon_{k k}}{k T}\right) \frac{\sigma_{i i}^{3} \sigma_{j j}^{3} \sigma_{k k}^{3}}{\sigma_{i j} \sigma_{i k} \sigma_{j k}} n_{p i} n_{p j} n_{p k} \mu_{i}^{* 2} \mu_{j}^{* 2} \mu_{k}^{*}\right.
$$$$
+\sum_{i} \sum_{j} \sum_{g} x_{i} x_{j} x_{g}\left(\frac{\epsilon_{i i}}{k T}\right)\left(\frac{\epsilon_{j j}}{k T}\right)\left(\frac{\epsilon_{g g}}{k T}\right) \frac{\sigma_{i i}^{3} \sigma_{j j}^{3} \sigma_{g g}^{3}}{\sigma_{i j} \sigma_{i g} \sigma_{j g}} n_{p i} n_{p j} n_{p g} \mu_{i}^{* 2} \mu_{j}^{* 2} \mu_{g}^{* 2} I_{\Xi}
$$ 


$$
I_{3, i j g, x_{k}}=\sum_{n=0}^{4} n c_{n, i j g} \eta^{n-1} \zeta_{3, x_{k}}
$$

\section{Enthalpy and entropy}

$$
\frac{h^{r e s}}{R T}=-T\left[\frac{\partial \tilde{a}^{r e s}}{\partial T}\right]_{\rho, x_{i}}+Z-1
$$

$\frac{s^{r e s}(P, T)}{R}=-T\left[\left(\frac{\partial \tilde{a}^{r e s}}{\partial T}\right)_{\rho, x_{i}}+\frac{\tilde{a}^{r e s}}{T}\right]+\ln Z$

$\left(\frac{\partial \tilde{a}^{r e s}}{\partial T}\right)_{\rho, x_{i}}=\left(\frac{\partial \tilde{a}^{\text {chain }}}{\partial T}\right)_{\rho, x_{i}}+\left(\frac{\partial \tilde{a}^{\text {disp }}}{\partial T}\right)_{\rho, x_{i}}+\left(\frac{\partial \tilde{a}^{\text {polar }}}{\partial T}\right)_{\rho, x_{i}}$

$d_{i, T}=\frac{\partial d_{i}}{\partial T}=\sigma_{i}\left(3 \frac{\epsilon_{i}}{k T^{2}}\right)\left[-0.12 \exp \left(-3 \frac{\epsilon_{i}}{k T}\right)\right]$

$$
\zeta_{n, T}=\frac{\partial \zeta_{n}}{\partial T}=\frac{\pi}{6} \rho \sum_{i} x_{i} m_{i} n d_{i, T} d_{i}^{n-1} \quad n \in\{1,2,3\}
$$

$$
\left(\frac{\partial \tilde{a}^{\text {chain }}}{\partial T}\right)_{\rho, x_{i}}=\bar{m}\left(\frac{\partial \tilde{a}^{h s}}{\partial T}\right)_{\rho, x_{i}}-\sum_{i} x_{i}\left(m_{i}-1\right)\left(g_{i i}^{h s}\right)^{-1}\left(\frac{\partial g_{i i}^{h s}}{\partial T}\right)_{\rho, x_{i}}
$$

$$
\begin{aligned}
\left(\frac{\partial \tilde{a}^{h s}}{\partial T}\right)_{\rho, x_{i}}= & \frac{1}{\zeta_{0}}\left[\frac{3\left(\zeta_{1, T} \zeta_{2}+\zeta_{1} \zeta_{2, T}\right)}{\left(1-\zeta_{3}\right)}+\frac{3 \zeta_{1} \zeta_{2} \zeta_{3, T}}{\left(1-\zeta_{3}\right)^{2}}+\frac{3 \zeta_{2}^{2} \zeta_{2, T}}{\zeta_{3}\left(1-\zeta_{3}\right)^{2}}+\frac{\zeta_{2}^{3} \zeta_{3, T}\left(3 \zeta_{3}-1\right.}{\zeta_{3}^{2}\left(1-\zeta_{3}\right)^{3}}\right. \\
& \left.+\left(\frac{3 \zeta_{2}^{2} \zeta_{2, T} \zeta_{3}-2 \zeta_{2}^{3} \zeta_{3, T}}{\zeta_{3}^{3}}\right) \ln \left(1-\zeta_{3}\right)+\left(\zeta_{0}-\frac{\zeta_{2}^{3}}{\zeta_{3}^{2}}\right) \frac{\zeta_{3, T}}{\left(1-\zeta_{3}\right)}\right]
\end{aligned}
$$

$$
d_{i j}=\left(\frac{d_{i} d_{i}}{d_{i}+d_{i}}\right)
$$


$d_{i j, T}=\frac{d_{i, T} d_{j}+d_{i} d_{j, T}}{d_{i}+d_{j}}-\frac{d_{i} d_{j}\left(d_{i, T}+d_{j, T}\right)}{\left(d_{i}+d_{j}\right)^{2}}$

$\frac{\partial g_{i j}^{h s}}{\partial \mathrm{T}}$

$$
\begin{aligned}
& =\frac{\zeta_{3, T}}{\left(1-\zeta_{3}\right)^{2}}+\left(d_{i j, T}\right) \frac{3 \zeta_{2}}{\left(1-\zeta_{3}\right)^{2}}+\left(d_{i j}\right)\left(\frac{3 \zeta_{2, T}}{\left(1-\zeta_{3}\right)^{2}}+\frac{6 \zeta_{2} \zeta_{3, T}}{\left(1-\zeta_{3}\right)^{3}}\right)+(\mathrm{S} 72 \\
& \frac{2 \zeta_{2}^{2}}{\left(1-\zeta_{3}\right)^{3}}+\left(d_{i j}\right)^{2}\left(\frac{4 \zeta_{2} \zeta_{2, T}}{\left(1-\zeta_{3}\right)^{3}}+\frac{6 \zeta_{2}^{2} \zeta_{3, T}}{\left(1-\zeta_{3}\right)^{4}}\right)
\end{aligned}
$$

$\left(\frac{\partial \tilde{a}^{d i s p}}{\partial \mathrm{T}}\right)_{\rho, x_{i}}=-2 \pi \rho\left(\frac{\partial I_{1}}{\partial \mathrm{T}}-\frac{I_{1}}{\mathrm{~T}}\right) \overline{m^{2} \epsilon \sigma^{3}}-\pi \rho \bar{m}\left[\frac{\partial C_{1}}{\partial \mathrm{T}} I_{2}+C_{1} \frac{\partial I_{2}}{\partial \mathrm{T}}-2 C_{1} \frac{I_{2}}{\mathrm{~T}}\right] \overline{m^{2} \epsilon^{2} \sigma^{3}}(\mathrm{~S} 73$

$\frac{\partial I_{1}}{\partial \mathrm{T}}=\sum_{i=0}^{6} a_{i}(\bar{m}) i \zeta_{3, T} \eta^{i-1}$

$\frac{\partial I_{2}}{\partial \mathrm{T}}=\sum_{i=0}^{6} b_{i}(\bar{m}) i \zeta_{3, T} \eta^{i-1}$

$\frac{\partial C_{1}}{\partial \mathrm{T}}=\zeta_{3, T} C_{2}$

$\left(\frac{\partial \tilde{a}^{\text {polar }}}{\partial \mathrm{T}}\right)_{\rho, x_{i}}=\frac{\tilde{a}_{2}\left(\tilde{a}_{2, T}+\tilde{a}_{3, T}\right)-2 \tilde{a}_{2, T} \tilde{a}_{3}}{\tilde{a}_{2}\left(1-\tilde{a}_{3} / \tilde{a}_{2}\right)^{2}}$

$\tilde{a}_{2, T}=-\pi \rho \sum_{i} \sum_{j} x_{i} x_{j} \frac{\epsilon_{i i} \epsilon_{j j} \sigma_{i i}^{3} \sigma_{j j}^{3}}{k T k T \sigma_{i j}^{3}} n_{p i} n_{p j} \mu_{i}^{* 2} \mu_{j}^{* 2}\left(I_{2, i j, T}-\frac{2}{T} I_{2, i j}\right)$

$\tilde{a}_{3, T}$

$$
\begin{aligned}
& =-\frac{4 \pi^{2}}{3} \rho^{2} \sum_{i} \sum_{j} \sum_{k} x_{i} x_{j} x_{k} \frac{\epsilon_{i i} \epsilon_{j j} \epsilon_{k k} \sigma_{i i}^{3} \sigma_{j j}^{3} \sigma_{k k}^{3}}{k k T k T \sigma_{i j} \sigma_{i k} \sigma_{j k}} n_{p i} n_{p j} n_{p k} \mu_{i}^{* 2} \mu_{j}^{*} \\
& \left(I_{3, i j k, T}-\frac{3}{T} I_{3, i j k}\right)
\end{aligned}
$$




$$
\begin{aligned}
I_{2, i j, T} & =\sum_{n=0}^{4}\left[n\left(a_{n, i j}+b_{n, i j} \frac{\epsilon_{i j}}{k T}\right) \eta^{n-1} \zeta_{3, T}-\eta^{n} b_{n, i j} \frac{\epsilon_{i j}}{k T^{2}}\right] \\
I_{3, i j k, T} & =\sum_{n=0}^{4} n c_{n, i j k} \eta^{n-1} \zeta_{3, T}
\end{aligned}
$$

